\title{
Robust Stability Analysis for the New Type Rural Social Endowment Insurance System with Minor Fluctuations in China
}

\author{
Lijian Wang \\ School of Public Policy and Administration, Xi'an Jiaotong University, Xi'an 710049, China \\ Correspondence should be addressed to Lijian Wang, wanglijian2@163.com
}

Received 27 April 2012; Revised 16 July 2012; Accepted 17 July 2012

Academic Editor: Victor S. Kozyakin

Copyright (C) 2012 Lijian Wang. This is an open access article distributed under the Creative Commons Attribution License, which permits unrestricted use, distribution, and reproduction in any medium, provided the original work is properly cited.

The new type rural social endowment insurance system is one of the important policy arrangements in public welfare field in China. Quantitative research is theoretically and practically important for studying the new type rural social endowment insurance system. In this paper, based on the modern control theory, the linear control model for the new type rural social endowment insurance system is set up, and then the robust stability for the new type rural social endowment insurance system of China with minor fluctuations is discussed by using the linear control model.

\section{Introduction}

With the rapid growing of the rural aging population, support demand of the elderly rural population has gradually increased in China. But in comparison to the improving pension insurance system for urban workers, construction of rural social endowment insurance has lagged far behind in the objective requirements of socioeconomic development [1]. Therefore, the State Council promulgated the document named "The Guidance of Launching the New Type Rural Social Endowment Insurance" in 2009, which defines the content and objective of the China's new type rural social endowment insurance system. The new type rural social endowment insurance system has been warmly welcomed and supported by the local government and vast peasantry since pilot work was carryied out in China. By June 2011, nine provinces (municipalities and autonomous regions) had realized the full coverage of new type rural social endowment insurance system, and pilot work has made remarkable achievement. In response to the appeal of prime minister Wen Jiabao, that is, realized the full coverage of new type rural social endowment insurance system in his term. Governments 
around the world has been speeding up the construction process of the new type rural social endowment insurance system in practice.

166 countries have established a pension insurance system in the world. But the patterns of old-age insurance are various, particularly between the developing countries and developed countries [2]. Although the patterns are different, they all offer a better pension protection for farmers in their countries. The experience of endowment insurance in developed countries give us much valuable apocalypse, including recognizing the basic target of the endowment insurance system, intensifying the responsibility of the government in the system, pushing forward this system into legalization and insisting on the principle of multilayer [3-5], which has an important reference meaning for choosing a suitable endowment insurance mode in our country.

From the new type rural social endowment insurance policies, we can find the following messages.

(I) The new type rural social endowment insurance funds should be made of individual contributions, collective benefits, and government subsidies. (i) The payments of the rural individual person: the individual contributions of the new type rural social endowment insurance, unlike the poor social assistance and social relief system, is a prerequisite for the enjoyment of the treatment. Except for the rural residents who have already reached the age of 60 or older, currently individual contribution located five grades form 100 to 500 yuan and local governments can set up additional grades based on their actual need for choosing. (ii) The collective benefits: local governments insured people pay subsidies to facilitate the participants to mobilize the enthusiasm of the farmers to help the poor people insured and to increase the accumulation of individual accounts. Local government subsidies to the standard of not less than 30 yuan per person per year, the selection of a high standard of pay grade may be given appropriate incentives, right of persons with disabilities in rural areas with severe problems such as payment groups, local governments on their behalf to pay some or all of the minimum standards pension insurance. (iii) The financial subsidies: the central government granted a full subsidy for basic old-age pensions, in which the central government on the central and western regions by the central authorities were given a full basic pension subsidies, subsidies for the east half.

(II) Pension entitlements of the new type rural social endowment insurance are from the basic pension and individual pension accounts. (i) Basic pension: central government determines a basic pension standard of 55 yuan per person per month, local governments can improve the standard on the basis of their own conditions. According to the economic development and price changes, central government adjust the level of basic pensions. (ii) Individual pension accounts: the issued standard is the total storage of individual pension account divided by 139. For example, if an individual chooses the grade of 300 yuan and the local government subsidize 50 yuan per year, paying for 30 years with an average annual interest rate of $3 \%$, the cumulative storage of personal accounts amounted to 16,651 yuan when he reaches 60. Divided by 139, the monthly pensions in personal accounts are 120 .

Fund raising standard and the level of pension issuance are both crucial problems of the system designation during the pilot. A recent survey suggests that at some pilot counties, there are some differences between fund raising standard, parameter setting of the issuance of pension, and the expectation of the local residents. Therefore, before an all round supporting 
system, in order to ensure and promote the sustainable development of the new type rural social endowment insurance system, it is necessary to study on the mathematical model and stability analysis for the new type rural social endowment insurance system.

Many scholars have noticed this problem, but existing literature mainly focuse on the need for the new type rural social endowment insurance, the existing problems, and countermeasures, and so on [6,7]. From modern control theory perspective, the existing literature rarely quantitatively analyzed the new type rural social endowment insurance and the papers from macroscopical perspective on the mathematical model and stability analysis of the new type rural social pension insurance system are even rare.

The use of modern control theory has a certain predominance to explore the mathematical model and stability analysis of the new type rural social pension insurance system. Oliveiraa introduces some conditions for robust stability with continuously change [8]. Qiu et al. constructs an asymptotically stabilizing controller based on Lyapunov function under polar coordinates [9]. According to eigenvalue criterion, the robust stability necessary and sufficient conditions of single-multiplication interval linear systems are given by Qiu et al. [10], Lakshmanan et al. [11], and Akbari Moornani and Haeri [12] . The research has got some progress by applying differential equations theory to study the problem of social security systems. In 2006, the author established the nonlinear model of social security systems and researched the sustainable problem, established the linear model of social security systems and studied the reliability problem, and studied the sustainable problem by establishing linear model of social security systems and applying the modern control theory.

This paper, based on the modern theory of social security systems combining with related mathematical methods and control theory, sets up a differential equations model to quantitatively analyze the relations among the payments of the rural individual person, collective benefits, and financial subsidies. Finally, we analyze the robust stability of the new type rural social endowment insurance system.

\section{Basic Hypotheses}

The new type rural social endowment insurance is viewed as a crucial ingredient of Rural social security system. It is of great importance to perfect rural social endowment insurance for the purpose of propelling the development of Chinese rural economy and even the development of the whole society. The most important feature of new type rural social endowment insurance is to give financial subsidies and collective subsidies. As a result, the new-type rural social endowment insurance fund comes from the payments of the rural population, the collective benefits, and the financial subsidies. The new type rural social endowment insurance fund pensions come from the basic pension account and individual saving accounts. The structure diagram for the incomings and outgoings of new type rural social endowment insurance fund can be described in Figure 1.

A recent survey suggests that at some pilot counties, there are some differences between fund raising standard, parameter setting of the issuance of pension, and the expectation of the local residents. Therefore before an all round supporting system, it is necessary to study how to raise money that cannot only be afforded by the rural people but also guarantee their basic requirement, so we can comprehensively push forward our new rural social pension and achieve the goal of caring for the old. This paper mainly focuses on the optimal control problem for the new type rural social endowment insurance, in order to set an optimum configuration of the payments of the rural population, the collective benefits, 


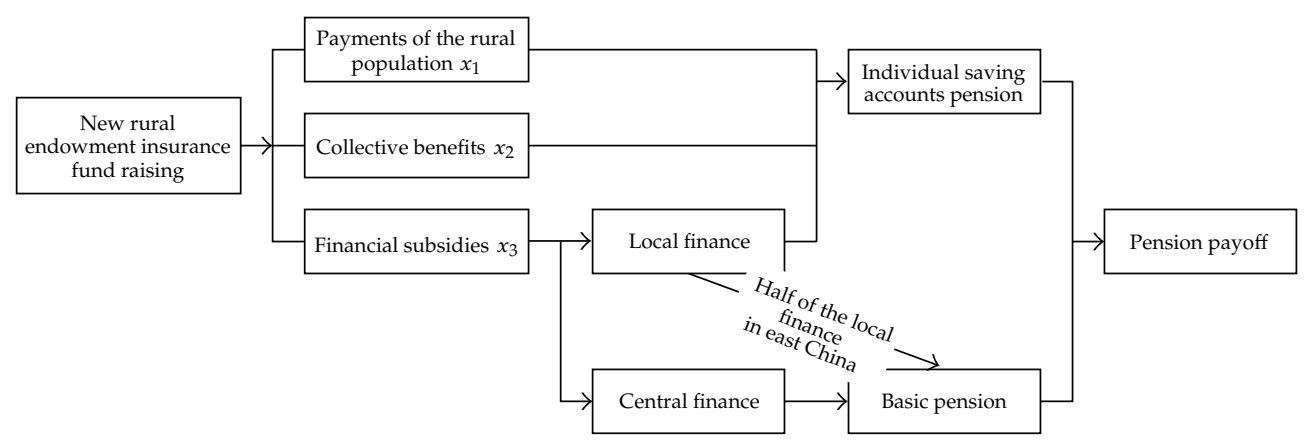

Figure 1: Incomings and outgoings of new-type rural social endowment insurance fund.

and the financial subsidies. Furthermore, the optimum configuration affects the enjoyment of pensions, the research necessary for this dissertation is satisfied.

Let $x_{1}, x_{2}, x_{3}, x_{4}$ denote the individual contribution average amount, the financial subsidies average amount, the collective benefits average amount, and the incremental funding of new type rural social endowment insurance, respectively, at time $t$, and let $x_{10}$, $x_{20}, x_{30}, x_{40}$ denote the steady value of $x_{1}, x_{2}, x_{3}, x_{4}$ at time $t_{0}$. According to "Regulations on the new rural social endowment insurance to carry out the pilot guidance" which is issued by the Chinese State Council and the relations between each complex element in the new rural social endowment insurance, we have the following basic hypotheses.

$\left(H_{1}\right)$ The pensions gap in individual contribution grades is very small, $x_{1}$ will decrease $a_{1} x_{1}$.

$\left(H_{2}\right)$ A part of financial subsidies go to personal accounts, this has a certain role to encourage the insured to pay pension premiums, $x_{1}$ will increase $a_{2} x_{2}$.

$\left(H_{3}\right)$ For balancing the new rural social endowment insurance system, financial subsidies will grow with individual contribution decreasing, the incremental value is $a_{1} x_{1}$.

$\left(H_{4}\right)$ In practice, the financial subsidies and the collective benefits have played alternating roles, one aspect wanes and the other waxes. We assume that the volume of change is $a_{3} x_{2}$.

$\left(H_{5}\right)$ The collective benefits depend on the village-level collective economy, and the local government plays a game with the authorities in decision making, so $x_{3}$ will decrease $a_{4} x_{3}$.

$\left(H_{6}\right)$ The premise of the new rural social endowment insurance system is optimum running, at time $t$, if $x_{4}(t)>0$, then $\left(d x_{4}(t) / d t\right)<0$; if $x_{4}(t)<0$, then $\left(d x_{4}(t) / d t\right)>$ 0 .

$\left(\mathrm{H}_{7}\right)$ With the aging of the rural population growing increasingly fast, especially after the enforcement of the family planning policy, the traditional family function is gradually blurred, and the rural endowment problem is getting worse, $x_{4}$ need to pick up, so $a_{i}>0(i=1,2, \ldots, 8)$.

$\left(H_{8}\right)$ In this paper, we assume that there were minor fluctuations in the parameters, so a linear model is used for the new rural social endowment insurance system. 
$a_{1}-a_{8}$ indicate the variation coefficient of the fluctuations of $x_{1}, x_{2}, x_{3}$, and $x_{4}$.

From $\mathrm{H}_{1}-\mathrm{H}_{8}$, we obtain

$$
\begin{aligned}
& \frac{d x_{1}}{d t}=-a_{1} x_{1}+a_{2} x_{2} \\
& \frac{d x_{2}}{d t}=a_{1} x_{1}-a_{3} x_{2} \\
& \frac{d x_{3}}{d t}=a_{3} x_{2}-a_{4} x_{3} \\
& \frac{d x_{4}}{d t}=a_{5} x_{1}+a_{6} x_{2}+a_{7} x_{3}-a_{8} x_{4}
\end{aligned}
$$

The matrix form of (2.1) is

$$
\dot{x}(t)=A x(t),
$$

where

$$
A=\left[\begin{array}{cccc}
-a_{1} & a_{2} & 0 & 0 \\
a_{1} & -a_{3} & 0 & 0 \\
0 & a_{3} & -a_{4} & 0 \\
a_{5} & a_{6} & a_{7} & -a_{8}
\end{array}\right], \quad x(t)=\left[\begin{array}{l}
x_{1} \\
x_{2} \\
x_{3} \\
x_{4}
\end{array}\right]
$$

The proper equations of (2.1) are

$$
|\lambda E-A|=\left|\begin{array}{cccc}
\lambda+a_{1} & -a_{2} & 0 & 0 \\
-a_{1} & \lambda+a_{3} & 0 & 0 \\
0 & -a_{3} & \lambda+a_{4} & 0 \\
-a_{5} & -a_{6} & -a_{7} & \lambda+a_{8}
\end{array}\right|=\left(\lambda+a_{4}\right)\left[\lambda^{2}+\left(a_{1}+a_{3}\right) \lambda+a_{1} a_{3}-a_{1} a_{2}\right]\left(\lambda+a_{8}\right)
$$

Let

$$
|\lambda E-A|=0
$$

we have

$$
\begin{aligned}
& \lambda_{1}=-a_{4}, \\
& \lambda_{2}=-\frac{a_{1}+a_{3}}{2}-\sqrt{\left(\frac{a_{1}+a_{3}}{2}\right)^{2}-\left(a_{1} a_{3}-a_{1} a_{2}\right)}, \\
& \lambda_{3}=-\frac{a_{1}+a_{3}}{2}+\sqrt{\left(\frac{a_{1}+a_{3}}{2}\right)^{2}-\left(a_{1} a_{3}-a_{1} a_{2}\right)}, \\
& \lambda_{4}=-a_{8} .
\end{aligned}
$$


On the grounds of the different natures of $\lambda_{1}, \lambda_{2}, \lambda_{3}$, and $\lambda_{4}$, we get the following solutions of (2.1):

$$
\begin{aligned}
& x_{1}(t)=c_{11} e^{\lambda_{1} t}+c_{12} e^{\lambda_{2} t}+c_{13} e^{\lambda_{3} t}+c_{14} e^{\lambda_{4} t}, \\
& x_{2}(t)=c_{21} e^{\lambda_{1} t}+c_{22} e^{\lambda_{2} t}+c_{23} e^{\lambda_{3} t}+c_{24} e^{\lambda_{4} t}, \\
& x_{3}(t)=c_{31} e^{\lambda_{1} t}+c_{32} e^{\lambda_{2} t}+c_{33} e^{\lambda_{3} t}+c_{34} e^{\lambda_{4} t}, \\
& x_{4}(t)=c_{41} e^{\lambda_{1} t}+c_{42} e^{\lambda_{2} t}+c_{43} e^{\lambda_{3} t}+c_{44} e^{\lambda_{4} t},
\end{aligned}
$$

where $c_{i j}(i, j=1,2,3,4)$ and $\lambda_{i}(i=1,2,3,4)$ are unknown constant numbers.

\section{Definition of System Stability}

Control Model.

$$
\begin{aligned}
& \dot{x}(t)=A(t) x(t)+B(t) u(t), \\
& y(t)=C(t) x(t)+D(t) u(t) .
\end{aligned}
$$

Its linear time-invariant system is as follows:

$$
\begin{aligned}
& \dot{x}(t)=A x(t)+B u(t), \\
& y(t)=C x(t)+D u(t) .
\end{aligned}
$$

Equations (2.1) show that $x_{2}$ exists in the total four equations. Through controlling the second equation, the accurate control of the equations is obtained. So, the control model of the new rural social endowment insurance system is as follows:

$$
\begin{aligned}
& \frac{d x_{1}}{d t}=-a_{1} x_{1}+a_{2} x_{2}, \\
& \frac{d x_{2}}{d t}=a_{1} x_{1}-a_{3} x_{2}+u_{1}, \\
& \frac{d x_{3}}{d t}=a_{3} x_{2}-a_{4} x_{3}, \\
& \frac{d x_{4}}{d t}=a_{5} x_{1}+a_{6} x_{2}+a_{7} x_{3}-a_{8} x_{4} .
\end{aligned}
$$

Matrix form of (3.3) is

$$
\dot{x}(t)=A x(t)+B u(t),
$$


where

$$
A=\left[\begin{array}{cccc}
-a_{1} & a_{2} & 0 & 0 \\
a_{1} & -a_{3} & 0 & 0 \\
0 & a_{3} & -a_{4} & 0 \\
a_{5} & a_{6} & a_{7} & -a_{8}
\end{array}\right] ; \quad u(t)=\left[\begin{array}{cccc}
0 & u_{1} & 0 & 0
\end{array}\right] ; \quad x(t)=\left[\begin{array}{c}
x_{1} \\
x_{2} \\
x_{3} \\
x_{4}
\end{array}\right] ; \quad B=\left[\begin{array}{l}
0 \\
1 \\
0 \\
0
\end{array}\right]
$$

Theorem 3.1. The sufficient and necessary condition of system (2.1) is stable when all of the characteristic value of A have negative real parts, and the characteristic value which has zero real parts is the simple root of its minimal polynomial. The sufficient and necessary condition asymptotically stable of system (2.1) is that all of the characteristic value of A have negative real parts [13].

For the new rural social endowment insurance system, we can judge the stability of the system by solving matrix A eigenvalues.

From $a_{i}>0(i=1,2, \ldots, 8)$, we have $\lambda_{1}<0, \lambda_{2}<0, \lambda_{4}<0$. If $a_{3}>a_{2}$, then $\lambda_{3}<0$. According to the direct algorithm for the stability analysis of the new rural social endowment insurance system, if $a_{3}>a_{2}$ and all of eigenvalues of $A$ have negative real parts or zero real parts, thus system (2.1) is stable. If $a_{3}>a_{2}$ and all of eigenvalues of $A$ have negative real parts, then system (2.1) is asymptotic stable.

\section{Robust Stability Analysis for the New Type Rural Social Endowment Insurance System}

The new rural social endowment insurance system has so many disturbance factors, such as the modeling error, system aging, and uncertain factors. This paper gives an example.

Take the linearly perturbed case as an example, this paper analyzes the robust stability of system (2.1).

The eigenvalue equation of system (2.1) is

$$
|\lambda E-A|=\left(\lambda+a_{4}\right)\left[\lambda^{2}+\left(a_{1}+a_{3}\right) \lambda+a_{1} a_{3}-a_{1} a_{2}\right]\left(\lambda+a_{8}\right) .
$$

Equation (2.1) is not relative to $a_{5}, a_{6}, a_{7}$, so we take no account of the disturbance of $a_{5}, a_{6}, a_{7}$; we only consider the disturbance of the eigenvalue equation's diagonal element, such as

$$
\Delta A=\left|\begin{array}{cccc}
\varepsilon_{1} & 0 & 0 & 0 \\
0 & \varepsilon_{2} & 0 & 0 \\
0 & 0 & \varepsilon_{3} & 0 \\
0 & 0 & 0 & \varepsilon_{4}
\end{array}\right| .
$$

The linear perturbed system is

$$
\dot{x}(t)=A^{\prime} x(t),
$$

where $A^{\prime}=A+\Delta A$. 
The eigenvalue equation of system (4.3) is

$$
\begin{gathered}
\left|\lambda E-A^{\prime}\right|=\left|\begin{array}{cccc}
\lambda+a_{1}-\varepsilon_{1} & -a_{2} & 0 & 0 \\
-a_{1} & \lambda+a_{3}-\varepsilon_{2} & 0 & 0 \\
0 & -a_{3} & \lambda+a_{4}-\varepsilon_{3} & 0 \\
-a_{5} & -a_{6} & -a_{7} & \lambda+a_{8}-\varepsilon_{4}
\end{array}\right|=\left(\lambda+a_{4}-\varepsilon_{3}\right) \\
{\left[\lambda^{2}+\left(a_{1}-\varepsilon_{1}+a_{3}-\varepsilon_{2}\right) \lambda+\left(a_{1}-\varepsilon_{1}\right)\left(a_{3}-\varepsilon_{2}\right)-\left(a_{1}-\varepsilon_{1}\right) a_{2}\right]\left(\lambda+a_{8}-\varepsilon_{4}\right) .}
\end{gathered}
$$

By solving the eigenvalue equation, we obtain

$$
\begin{aligned}
& \lambda_{1}^{\prime}=-a_{4}+\varepsilon_{3} \\
& \lambda_{2}^{\prime}=-\frac{a_{1}-\varepsilon_{1}+a_{3}-\varepsilon_{2}}{2}-\sqrt{\left(\frac{a_{1}-\varepsilon_{1}+a_{3}-\varepsilon_{2}}{2}\right)^{2}-\left(a_{1}-\varepsilon_{1}\right)\left(a_{3}-\varepsilon_{2}\right)+\left(a_{1}-\varepsilon_{1}\right) a_{2}} \\
& \lambda_{3}^{\prime}=-\frac{a_{1}-\varepsilon_{1}+a_{3}-\varepsilon_{2}}{2}+\sqrt{\left(\frac{a_{1}-\varepsilon_{1}+a_{3}-\varepsilon_{2}}{2}\right)^{2}-\left(a_{1}-\varepsilon_{1}\right)\left(a_{3}-\varepsilon_{2}\right)+\left(a_{1}-\varepsilon_{1}\right) a_{2}} \\
& \lambda_{4}^{\prime}=-a_{8}+\varepsilon_{4} .
\end{aligned}
$$

Thus, if $\varepsilon_{3}<a_{4}, \varepsilon_{4}<a_{8},\left(\varepsilon_{1}+\varepsilon_{2}\right)<\left(a_{1}+a_{3}\right), \varepsilon_{1}<a_{1}, \varepsilon_{2}<\left(a_{3}-a_{2}\right)$, then $\lambda_{1}^{\prime}<0$, $\lambda_{2}^{\prime}<0, \lambda_{4}^{\prime}<0, \lambda_{3}^{\prime}<0$. Considering the minor fluctuations of the parameters, $\varepsilon_{3}<a_{4}, \varepsilon_{4}<a_{8}$, $\left(\varepsilon_{1}+\varepsilon_{2}\right)<\left(a_{1}+a_{3}\right)$ and $\varepsilon_{1}<a_{1}$ are true. So long as $\varepsilon_{2}<\left(a_{3}-a_{2}\right)$, we have $\lambda_{1}^{\prime}<0, \lambda_{2}^{\prime}<0$, $\lambda_{14}^{\prime}<0, \lambda_{3}^{\prime}<0$.

According to Theorem 3.1, if $\varepsilon_{2}<\left(a_{3}-a_{2}\right)$ and all of the eigenvalues of $A$ have negative real parts or zero real parts, then system (4.3) is stable. Furthermore, if $\varepsilon_{2}<\left(a_{3}-a_{2}\right)$ and all of the eigenvalues of $A$ have negative real parts, then system (4.3) is asymptotic stable. Therefore, if $\varepsilon_{3}<a_{4}, \varepsilon_{4}<a_{8},\left(\varepsilon_{1}+\varepsilon_{2}\right)<\left(a_{1}+a_{3}\right), \varepsilon_{1}<a_{1}, \varepsilon_{2}<\left(a_{3}-a_{2}\right)$, then system (2.1) is robust stable.

\section{Conclusions}

The tentative launch of the new type rural social endowment system marks significant progress during developing the security system for elderly care in rural China. In order to further perfecting the new type rural social endowment system, in this paper, based on the modern control theory, the linear control model for the new type rural social endowment insurance system is set up, and then the robust stability for the new type rural social endowment insurance system of China with minor fluctuations is discussed by using the linear control model.

This paper finds out that if $a_{3}>a_{2}$ and all of eigenvalues of $A$ have negative real parts, then system (2.1) is asymptotic stable; if $\varepsilon_{3}<a_{4}, \varepsilon_{4}<a_{8},\left(\varepsilon_{1}+\varepsilon_{2}\right)<\left(a_{1}+a_{3}\right), \varepsilon_{1}<a_{1}$, $\varepsilon_{2}<\left(a_{3}-a_{2}\right)$, then system $(2.1)$ is robust stable. 


\section{Acknowledgment}

This work was supported by the National Social Science Foundation of China Grant no. 09\&ZD057 and Ph.D. students' fund from MOE.

\section{References}

[1] A. Ebenstein and S. Leung, "Son preference and access to social insurance: evidence from China's rural pension program," Population and Development Review, vol. 36, no. 1, pp. 47-70, 2010.

[2] H. P. Van Dalen, K. Henkens, and D. A. Hershey, "Perceptions and expectations of pension savings adequacy: a comparative study of Dutch and American workers," Ageing and Society, vol. 30, no. 5, pp. 731-754, 2010.

[3] E. Fan, "Who benefits from public old age pensions? Evidence from a targeted program," Economic Development and Cultural Change, vol. 58, no. 2, pp. 297-322, 2010.

[4] E. Calvo, F. M. Bertranou, and E. Bertranou, "Are old-age pension system reforms moving away from individual retirement accounts in Latin America?" Journal of Social Policy, vol. 39, no. 2, pp. 223-234, 2010.

[5] E. Overbye, "Extending social security in developing countries: a review of three main strategies," International Journal of Social Welfare, vol. 14, no. 4, pp. 305-314, 2005.

[6] C. Shen and J. B. Williamson, "China's new rural pension scheme: can it be improved?" International Journal of Sociology and Social Policy, vol. 30, no. 5-6, pp. 239-250, 2010.

[7] Y. Yang, J. B. Williamson, and C. Shen, "Social security for China's rural aged: a proposal based on a universal non-contributory pension," International Journal of Social Welfare, vol. 19, no. 2, pp. 236-245, 2010.

[8] M. C. De Oliveira, J. Bernussou, and J. C. Geromel, "A new discrete-time robust stability condition," Systems and Control Letters, vol. 37, no. 4, pp. 261-265, 1999.

[9] F. Qiu, B. Cui, and Y. Ji, "Further results on robust stability of neutral system with mixed time-varying delays and nonlinear perturbations," Nonlinear Analysis: Real World Applications, vol. 11, no. 2, pp. 895-906, 2010.

[10] J. Q. Qiu, J. Chen, P. Shi, and H. J. Yang, “New stochastic robust stability criteria for time-varying delay neutral system with Markavian jump parameters," International Journal of Control, Automation and Systems, vol. 8, no. 2, pp. 418-424, 2010.

[11] S. Lakshmanan, T. Senthilkumar, and P. Balasubramaniam, "Improved results on robust stability of neutral systems with mixed time-varying delays and nonlinear perturbations," Applied Mathematical Modelling, vol. 35, no. 11, pp. 5355-5368, 2011.

[12] K. Akbari Moornani and M. Haeri, “On robust stability of LTI fractional-order delay systems of retarded and neutral type," Automatica, vol. 46, no. 2, pp. 362-368, 2010.

[13] Z. Du, Q. Zhang, and L. Liu, "New delay-dependent robust stability of discrete singular systems with time-varying delay," Asian Journal of Control, vol. 13, no. 1, pp. 136-147, 2011. 


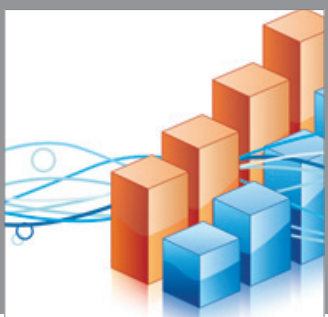

Advances in

Operations Research

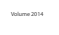

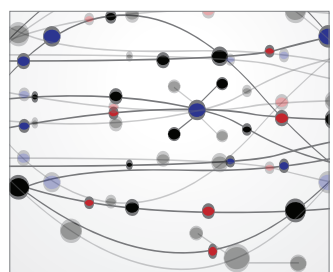

\section{The Scientific} World Journal
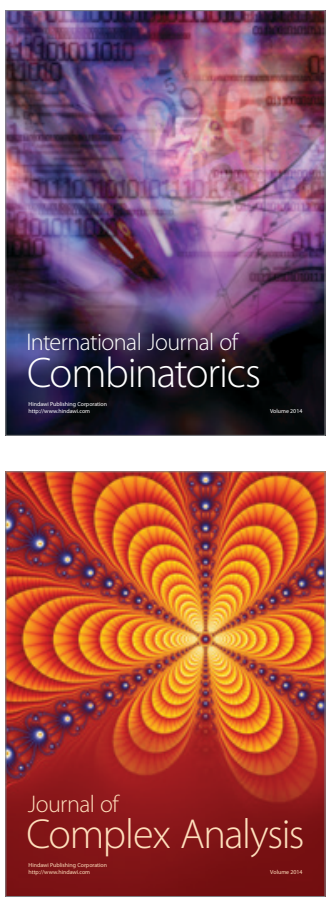

International Journal of

Mathematics and

Mathematical

Sciences
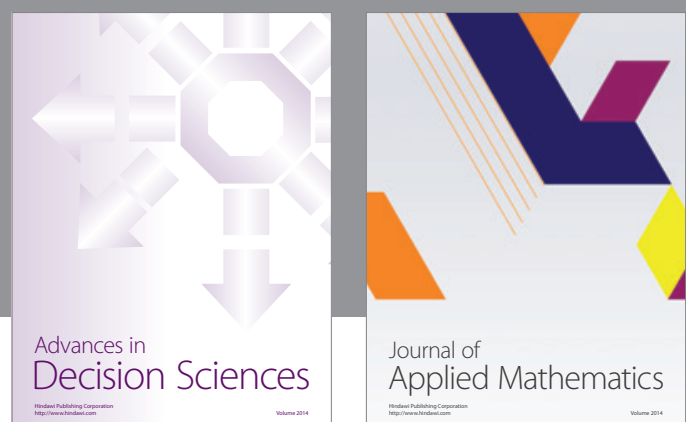

Journal of

Applied Mathematics
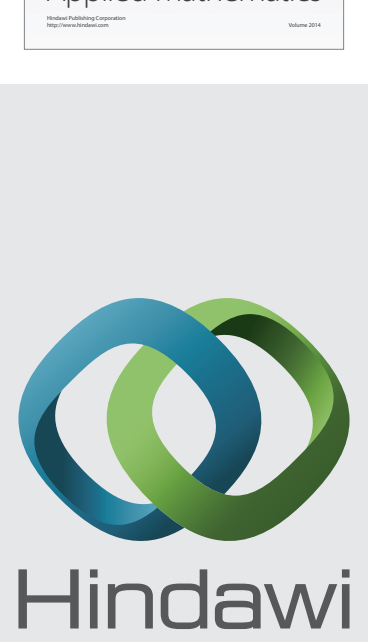

Submit your manuscripts at http://www.hindawi.com
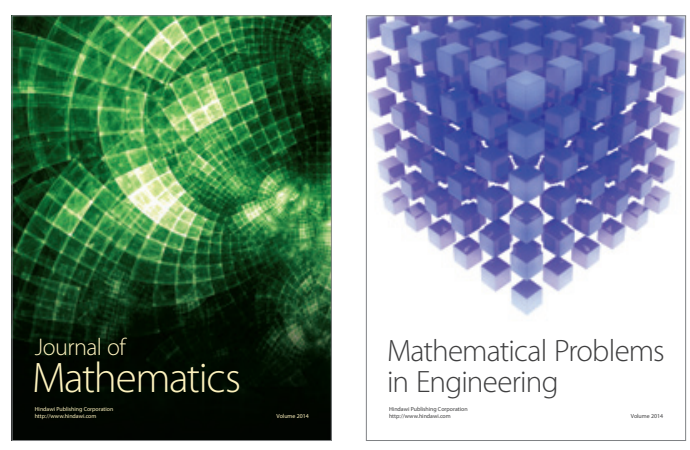

Mathematical Problems in Engineering
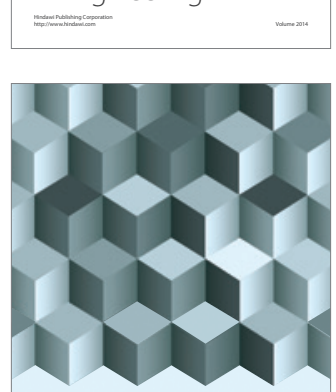

Journal of

Function Spaces
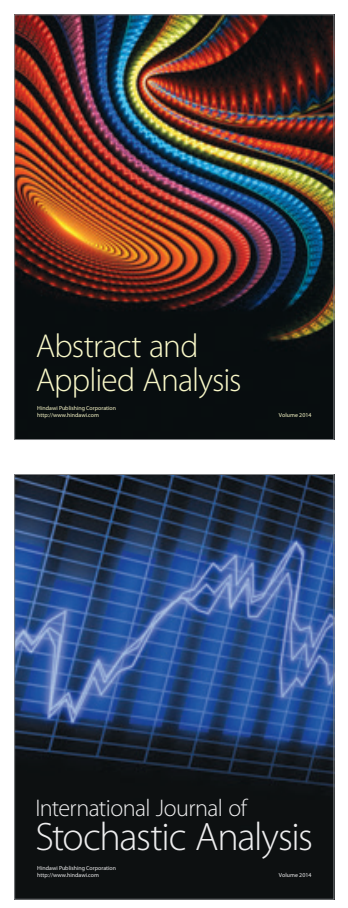

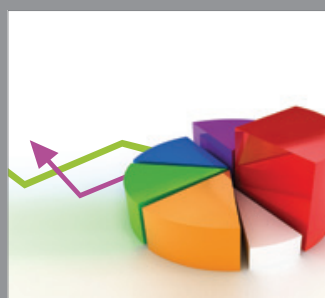

ournal of

Probability and Statistics

Promensencen
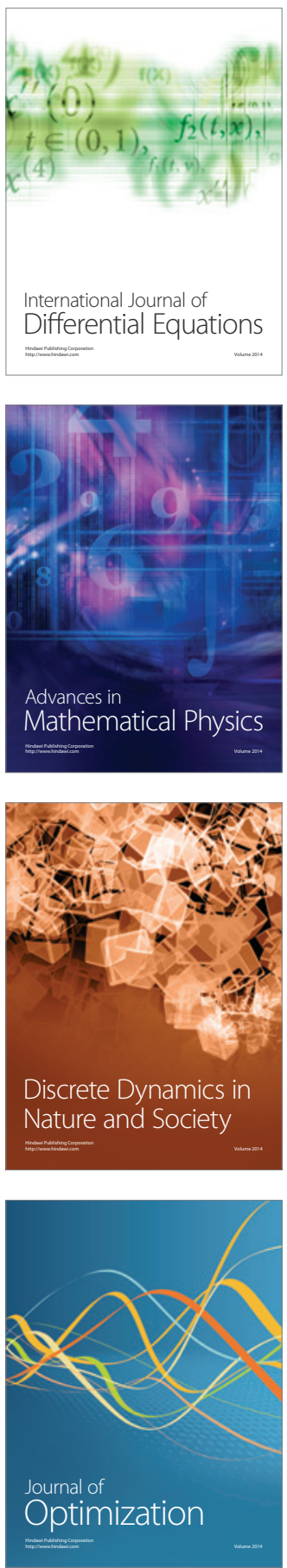